\title{
Hydrogen Sensors Made on InP or GaN with Electrophoretically Deposited Pd or Pt Nanoparticles
}

\author{
K. Zdansky ${ }^{a, *}$, O. Cernohorsky ${ }^{a, b}$ And R. YAtskiv ${ }^{a}$ \\ ${ }^{a}$ Institute of Photonics and Electronics, Academy of Sciences, Prague, Czech Republic \\ ${ }^{b}$ Faculty of Nuclear Sciences, Czech Technical University, Prague, Czech Republic
}

\begin{abstract}
High quality Schottky diodes were prepared by printing colloidal graphite on the polished wafers of $n$-type InP or $n$-type GaN. The wafers were earlier sparsely covered with palladium or platinum nanoparticles by electrophoresis from prepared colloid solutions in isooctane. Deposited contacts and nanoparticles were observed by scanning electron mictroscopy. Current voltage characteristics of the Schottky diodes showed high rectification ratios and the barrier heights close to the value of vacuum-level-alignment of the Schottky-Mott limit. The sensitivity to hydrogen of the diodes was measured in the flow of hydrogen/nitrogen mixtures of various hydrogen concentrations in the range from $1 \mathrm{ppm}$ to $1000 \mathrm{ppm}$. The estimated detection limits of the diodes were in the sub-ppm range. The diodes represent orders-of-magnitude improvement over the best hydrogen sensors reported previously.
\end{abstract}

PACS: 82.45.Qr, 81.16.Hc, 73.61.Ey, 73.40.Kp, 73.40.Ei

\section{Introduction}

Hydrogen is used in many places in industry, medicine and research laboratories [1] where hydrogen sensors are used for safety reason. In addition, a hydrogen-sensitive sensor has a good application in apparatuses for finding small leaks in various closed systems, e.g. in equipments with a high or ultrahigh vacuum. Until recently, the only facility for that purpose was a helium leak detector, which is very costly and which uses helium, whose natural reserves are severely declining and its price rising [2]. Cheaper devices containing hydrogen sensors, using forming gas, which is a non-flammable mixture of hydrogen with nitrogen, can perform the same function.

Hydrogen sensors have been prepared on more kinds of semiconductors by using catalytic metals either in the gate of semiconductor-metal-semiconductor structures or as metal contacts of the Schottky barriers [3-15]. In those sensors, hydrogen molecules are dissociated by catalytic metals and hydrogen atoms form a dipole layer, which reduces effective work function of the metal and changes the current-voltage characteristics of the sensor.

In this article we bring our research report on the Schottky barriers on InP and GaN for the detection of hydrogen. InP is suitable for the detection of hydrogen at normal temperature, whereas GaN can be used at high temperatures up to $800^{\circ} \mathrm{C}$ [16]. For the dissociation of hydrogen molecules, we used nanoparticles of the catalytic metal Pd or Pt. An important difference from

\footnotetext{
* corresponding author; e-mail: zdansky@ufe.cz
}

other similar sensors is that the Schottky barrier is not formed only by catalytic metal nanoparticles, but also by conducting porous graphite. Layers of porous graphite form the Schottky barrier of the height value close to the vacuum-level alignment (the Schottky-Mott limit) [17], which is a good pre-requisite for high detection sensitivity, and they also allow free access of hydrogen gas to the semiconductor interface. The article closely relates to our previous studies published recently [18-20].

\section{Experimental}

Hydrogen sensors were made on professionally grown and polished semiconductor wafers. InP wafers were purchased from the company Wafer Technology, UK and GaN wafers were purchased from the company Kyma Technologies, USA. Dimensions of the wafers were $10 \times$ $10 \mathrm{~mm}^{2}$ and thickness $460 \mu \mathrm{m}$. The crystal orientation of cubic InP wafers was [110] and hexagonal GaN wafers were perpendicular to the $c$-axis. Polished surface of GaN wafers, on which the Schottky barriers were made, was the surface of $\mathrm{Ga}$ atoms. The wafers were $n$-type conductivity with doping density $10^{16} \mathrm{~cm}^{-3}$.

The wafers were electrophoretically deposited with catalytic metal nanoparticles, $\mathrm{Pd}$ or $\mathrm{Pt}$ from colloidal solutions. To this purpose, colloidal solutions of metal nanoparticles in isooctane were prepared in advance by reducing aqueous solutions of metal salts in reverse micelles with the surfactant sodium di-2ethylhexylsulfosuccinate (AOT) [21]. The aqueous solution of hydrazine was used as a reducing agent. The chemicals were purchased from the company SigmaAldrich. The shape and size of metal nanoparticles in 
the colloid was monitored by the scanning electron microscope JEOL JSM-7500F (SEM). Optical absorption due to the surface plasmon resonance of metal nanoparticles in the colloid was observed by the split-beam UV-VIS spectrophotometer SPECORD 210 of Analytic Jena.

Just before starting the electrophoresis, the wafers were treated in hot methanol. A wafer was provided with ohmic contact on one side and fixed by colloidal silver paint to the negative electrode (cathode) in the electrophoretic cell [22], so that the opposite plane-parallel graphite electrode was $1 \mathrm{~mm}$ far from the plain side of the wafer. The cell was washed several times with pure isooctane, filled with isooctane and the current through the cell was tested with the bias voltage $1000 \mathrm{~V}$. When the testing current was minimized, the cell was filled with the colloid solution containing catalytic metal nanoparticles and the electrophoresis was started by switching-on a keying voltage. The voltage $100 \mathrm{~V}$ was keyed periodically $50 \mathrm{~ms}$ on and $50 \mathrm{~ms}$ off. Periodically keyed voltage was chosen to improve deposition homogeneity and to eliminate deposition of heavier clustered nanoparticles [23]. The voltage was held on for $1 \mathrm{~h}$, which was the optimum time for preparing hydrogen sensors by further processing. Then the cell was emptied of the colloid solution and immediately rinsed with isopropyl alcohol to prevent caking of the colloid in the sample.

Several separated small-area $\left(0.075 \mathrm{~mm}^{2}\right)$ spots were made by colloidal graphite on the wafer side previously deposited with catalytic-metal nanoparticles and left to dry at normal conditions. Dry spots formed the Schottky barriers with the semiconductor. Thus, the Schottky diodes, each having one end on a spot and the other on the whole area ohmic contact on the other side of the semiconductor wafer, were fabricated. It should be noted that sparsely covered metal nanoparticles on the wafer did not short-circuit the Schottky barriers.

The SEM was used to image deposited metal nanoparticles and graphite spots forming the Schottky barriers. Current-voltage characteristics of the Schottky diodes and their dependences on hydrogen exposure were measured in the range of $10^{-14}-10^{-2}$ A by Keithley Source-Measure Unit 236 controlled by a computer using the software written in LabView. The measured diodes were placed in a specially designed cell with a positioning spring for contacting graphite spots of the Schottky diodes. The cell had two openings, one for input of a flowing gas and the other for its free outlet. These were used for measuring the diodes in the flow of hydrogennitrogen mixtures of various ratios.

\section{Results}

The SEM image of the surface of GaN with $\mathrm{Pt}$ nanoparticles deposited electrophoretically can be seen in Fig. 1. It is well seen that the surface is not covered totally. Spherical light spots of about $7 \mathrm{~nm}$ diameter representing Pt nanoparticles are grouped together in larger islands, which were formed by growing mechanism during the deposition. We observed that there were fewer and larger islands on well polished surfaces than on rougher surfaces. On the other hand, there were no groups (aggregates) of $\mathrm{Pt}$ nanoparticles in the colloid as found by observing SEM images of colloid drops made on metallic substrates and evaporating the solvent - isooctane (not shown). Light spherical Pt nanoparticles in Fig. 1 contrast with the dark cloud around them. This cloud was probably formed by the surfactant AOT. It should be noted that there was a surplus of AOT in the colloid, so that observing wider cloud-layer is plausible.

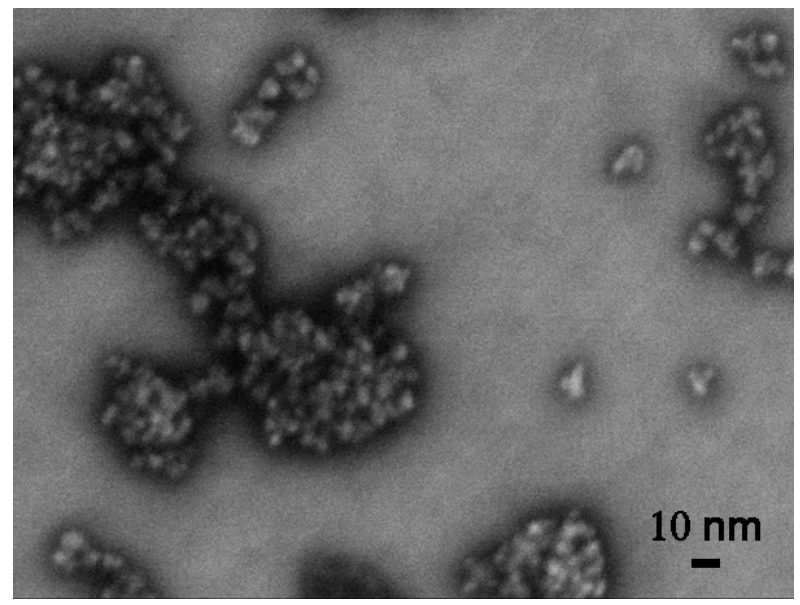

Fig. 1. SEM image of GaN surface with Pt nanoparticles.

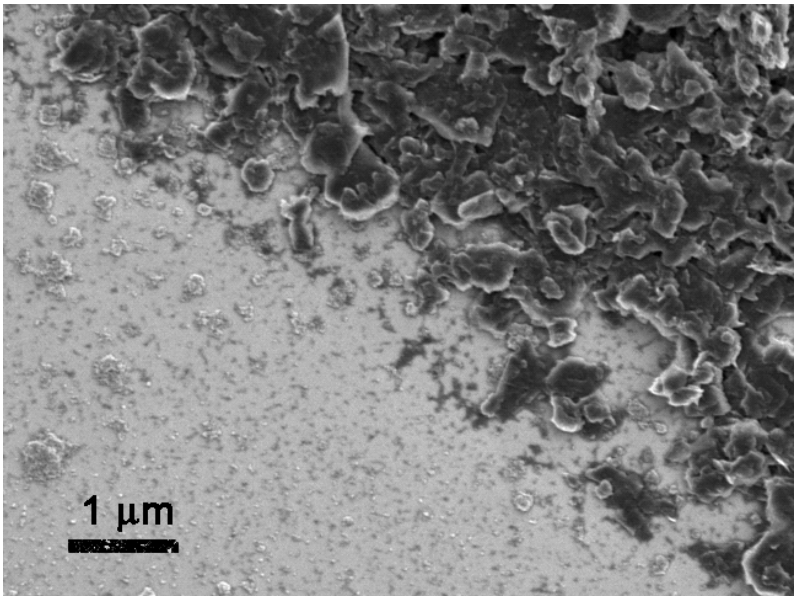

Fig. 2. SEM image of GaN surface with Pt nanoparticles at the edge of graphite contact.

Smaller magnification SEM image made on the same sample but at the edge of a graphite contact can be seen in Fig. 2. The graphite is seen in the upper-right part of the image and the lower-left part is the surface partly covered with Pt nanoparticles. Clearly, the graphite consists of particles with various sizes in the range $0.1-1 \mu \mathrm{m}$, piled in the layer of $20 \mu \mathrm{m}$ thickness as measured mechanically. 
At the edge line, dark graphite nanoparticles of the size less than $100 \mathrm{~nm}$ can be seen as they were formed during drying and shrinking of the colloidal graphite.

Similar SEM images as Figs. 1 and 2 were obtained in samples made on InP and in those with $\mathrm{Pd}$ nanoparticles.

Forward and reverse current-voltage characteristics of four Schottky diodes prepared on GaN and InP substrates with Pt and Pd nanoparticles are shown in Fig. 3. Forward and reverse characteristics are shown with the positive voltage scale. All diodes exhibit high rectification ratio, 8 orders at $1.5 \mathrm{~V}$ in the cases of $\mathrm{InP}$ based diodes and more than 12 orders at $2.5 \mathrm{~V}$ in the cases of GaN based diodes. Exact reverse currents of GaN based diodes could not be specified because they are smaller than the measurement limit of the apparatus, $10^{-14} \mathrm{~A}$. Leakage currents of $\mathrm{GaN}$ based diodes are at least three orders of magnitude smaller than leakage currents of InP based diodes which reflects the difference between the values of band gaps of these two semiconductors. The forward characteristics possess distinct linear parts in the semilog scale which enabled to estimate the values of the Schottky barrier heights; they were estimated at $0.87 \mathrm{eV}$ and $1.33 \mathrm{eV}$ for Pd nanoparticles deposited diodes on InP and GaN, respectively [20] and $0.92 \mathrm{eV}$ and $1.42 \mathrm{eV}$ for Pt diodes.

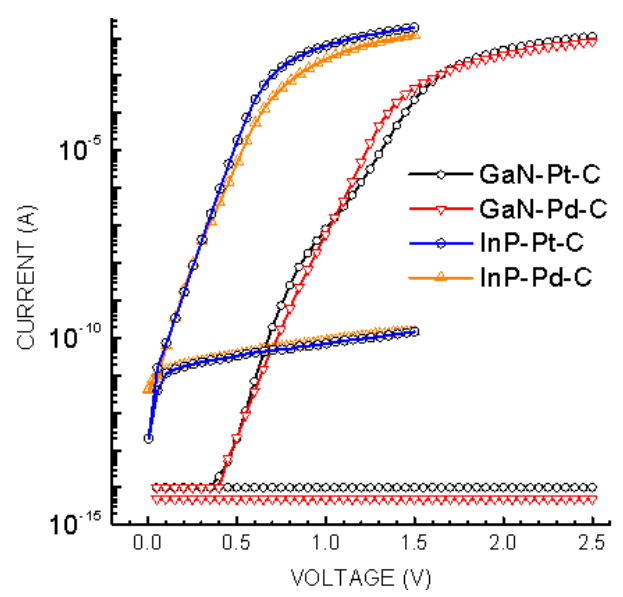

Fig. 3. Forward (up) and reverse (down) currentvoltage characteristics of four Schottky diodes prepared on GaN and InP wafers with Pt and Pd nanoparticles. Assignments are shown by the labels.

Current transients of the voltage biased diode InP-Pt-C upon exposure to the flow of five various $\mathrm{H}_{2} / \mathrm{N}_{2}$ mixtures with hydrogen concentrations, in the range from 1000 to 1 ppm, are shown in Fig. 4. The current increased to its maximum by more than six orders of magnitude with 1000 ppm mixture and to slightly smaller values with $165 \mathrm{ppm}$ and $30 \mathrm{ppm}$ mixtures. At lower concentrations, between $30 \mathrm{ppm}$ and $1 \mathrm{ppm}$, current maxima decreased substantially faster and the response time was much longer. Longer response time is particularly conspicuous with 1 ppm hydroben mixture which is shown by using a different scale on the top in Fig. 4.
TIME (s)

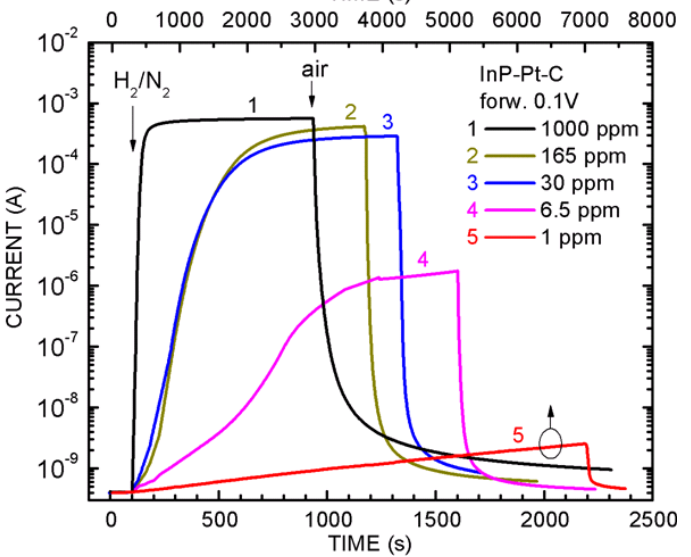

Fig. 4. Current transients of $0.1 \mathrm{~V}$ voltage biased diode InP-Pt-C upon exposure to flow of various hydrogennitrogen mixtures. Concentrations of hydrogen are shown by the labels. Top time scale holds for $1 \mathrm{ppm}$ curve.

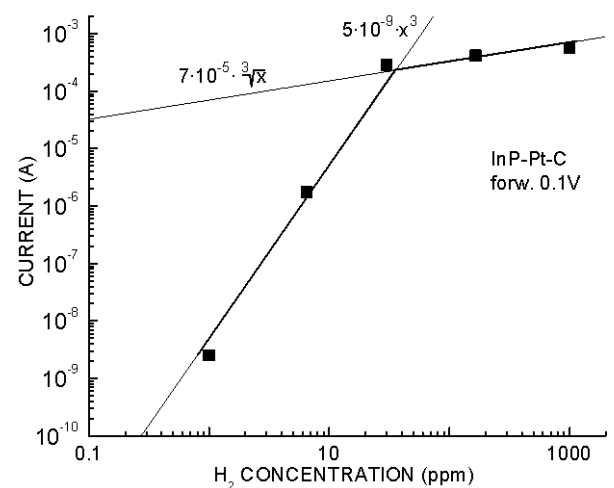

Fig. 5. The dependence of the maximum current of $0.1 \mathrm{~V}$ voltage biased $\mathrm{InP}-\mathrm{Pt}-\mathrm{C}$ diode on the hydrogen concentration.

The dependence of the maximum current of $0.1 \mathrm{~V}$ voltage biased diode $\mathrm{InP}-\mathrm{Pt}-\mathrm{C}$ on the hydrogen concentration is shown in Fig. 5. The dependence could be approximated with two straight lines in the semilog scale. The two straight lines represent two power dependences, with the cube root at higher hydrogen concentrations (1000$30 \mathrm{ppm}$ ) and with the cube power at lower concentrations (30-1 ppm).

\section{Discussion and conclusion}

We have shown that the Schottky diodes prepared by simple deposition of colloidal graphite on InP and GaN have an exceptionally high rectification ratio. The values of barrier heights estimated from the forward current voltage characteristics are close to vacuum level alignment between electron work function of the metal (5.12 eV for Pd [24] and 5.65 eV for Pt [25]) and electron affinity of the semiconductor $(4.38 \mathrm{eV}$ for InP [26] and 
$4.1 \mathrm{eV}$ for GaN [27]). The estimated values are larger than the level alignments for $\mathrm{Pd}$ on both $\mathrm{InP}(0.87 \mathrm{eV}$ $>0.74 \mathrm{eV})$ and GaN $(1.35 \mathrm{eV}>1.02 \mathrm{eV})$ and smaller for $\mathrm{Pt}$ on both $\operatorname{InP}(0.92 \mathrm{eV}<1.27 \mathrm{eV})$ and GaN $(1.42 \mathrm{eV}$ $<1.55 \mathrm{eV})$. It shows on a small density of interface states between the semiconductor and the metal in all types of our diodes leading to small Fermi level pinning. Consequently the diodes are highly sensitive to the electrical dipole layer at the interface, which reduces the effective work-function of the metal. Due to that and due to AOT enclosed catalytic metal nanoparticles and the porousness of graphite Schottky contacts, high sensitivity to hydrogen has been achieved. As a matter of fact, our diodes represent orders-of-magnitude improvement over the best hydrogen sensors reported previously [15]. We believe that the high-quality of the Schottky diodes is reached because the contacts formed by colloidal graphite and metal nanoparticles enclosed by AOT organic compound do not react chemically with InP or GaN semiconductors [28] and therefore no interface states are formed. We suggest that the same method of preparation should be successful for other semiconductors as well. We are going to investigate such kind of diodes on silicon which could lead to much cheaper gas sensors, compatible with sophisticated silicon technology.

\section{Acknowledgments}

We thank J. Zelinka from the Institute for helping with low-current measurements of the prepared diodes. We acknowledge financial support by COST Action MP0805, project OC10021 — "Study of metal nanoparticle layers deposited by electrophoresis on semiconductor III-V-N compounds" of the Ministry of Education of the Czech Republic, by project KAN401220801 — "Nanostructures of controlled size and dimensions" of the Academy of Sciences of the Czech Republic and by project 102/09/1037 - "Metallic nanolayers for semiconductor sensor and detector structures" of the Czech Science Foundation.

\section{References}

[1] C. Christofides, A. Mandelis, J. Appl. Phys. 68, 1 (1990).

[2] M. Block, in: 9th Europ. Conf. on Nondestructive Testing (ECNDT) Proc., Berlin, 2006, p. Tu.2.6.1; http://www.ndt.net/article/ecndt2006/doc/ Tu.2.6.1.pdf .

[3] M.C. Steele, B.A. Maclver, Appl. Phys. Lett. 28, 687 (1976).

[4] K. Ito, Surface Sci. 86, 345 (1979).

[5] P.F. Ruths, S. Ashok, S.J. Fonash, J.M. Ruths, IEEE Trans. Electron Dev. 28, 1003 (1981).
[6] M. Yousuf, B. Kuliyev, B. Lalevic, T.L. Poteat, Solid-State Electron. 25, 753 (1982).

[7] D.E. Aspenes, A. Heller, J. Vac. Sci. Technol. B 1 3, 602 (1983).

[8] L.M. Lechuga, A. Calle, D. Golmayo, P. Tejedor, F. Briones, Sens. Actuat. B 4, 515 (1991).

[9] H.I. Chen, Y.I Chou, C.Y. Chu, Sensors Actuat. B 85, $10(2002)$.

[10] K.W. Lin, H.I. Chen, H.M. Chuang, C.Y. Chen, C.T. Lu, C.C. Cheng, W.C. Liu, IEEE Sens. J. 4, 72 (2004).

[11] B.S. Kang, F. Ren, B.P. Gila, C.R. Abernathy, S.J. Pearton, Appl. Phys. Lett. 84, 1123 (2004).

[12] Y.I. Chou, C.M. Chen, W.C. Liu, H.I. Chen, IEEE Electron Dev. Lett. 26, 62 (2005).

[13] J.R. Huang, W.C. Hsu, H.I. Chen, W.C. Liu, Sens. Actuat. B 123, 1040 (2007).

[14] S.N. Das, A.K. Pal, J. Phys. D, Appl. Phys. 40, 7291 (2007).

[15] K. Skucha, Z. Fan, K. Jeon, A. Javey, B. Boser, Sens. Actuators B 145, 232 (2010).

[16] J.H. Song, W. Lu, J.S. Flynn, G.R. Brandes, Solid State Electron. 49, 1330 (2005).

[17] C. Tengstedt, W. Osikowicz, W.R. Salaneck, I.D. Parker, C.H. Hsu, M. Fahlman, Appl. Phys. Lett. 88, 053502 (2006).

[18] K. Zdansky, J. Zavadil, P. Kacerovsky, J. Lorincik, A. Fojtik, IEEE Trans. Nanotechnol. 9, 355 (2010).

[19] K. Zdansky, R. Yatskiv, J. Grym, O. Cernohorsky, J. Zavadil, F. Kostka, in: Proc. 2nd NANOCON Int. Conf., Olomouc (Czech Republic), 2010, Ed. R. Zboril, TANGER Ltd, Ostrava, 2010, p. 182.

[20] K. Zdansky, Nanoscale Res. Lett. 6, 490 (2011).

[21] D.H. Chen, J.J. Yeh, T.C. Huang, J. Colloid. Interf. Sci. 215, 159 (1999).

[22] K. Zdansky, J. Zavadil, P. Kacerovsky, J. Lorincik, J. Vanis, F. Kostka, O. Cernohorsky, A. Fojtik, J. Reboun, J. Cermak, Int. J. Mater. Res. (formerly Z. Metallkd.) 100, 1234 (2009).

[23] P. Gyftou, E.A. Pavlatou, N. Spyrellis, Appl. Surf. Sci. 254, 5910 (2008).

[24] http://environmentalchemistry.com/yogi/ periodic/Pd.html .

[25] http://environmentalchemistry.com/yogi/ periodic/Pt.html .

[26] http://www.ioffe.ru/SVA/NSM/Semicond/InP/ basic.html.

[27] http://www.ioffe.ru/SVA/NSM/Semicond/GaN/ basic.html.

[28] L.J. Brillson, H.L. Mosbacker, M.J. Hetzer, Y. Strzhemechny, D.C. Look, G. Cantwell, J. Zhang, J.J. Song, Appl. Surf. Sci. 254, 8000 (2008). 